\title{
THE MULTIPLICATORS OF CERTAIN SIMPLE GROUPS
}

\section{J. L. ALPERIN AND DANIEL GORENSTEIN ${ }^{1}$}

The recent paper of Steinberg [7] on the multiplicators of the finite simple groups of Lie type, the classical determination of the multiplicators of the alternating groups by Schur [6], a similar result of Janko for his group [3] and the (unpublished) work of J. G. Thompson on the Mathieu groups cover all but three families of known simple groups. In this paper we give a simple determination of the multiplicators for two of these families, namely the Suzuki groups and the Ree groups of characteristic three. Our results are well known for the Suzuki groups, with the exception of the one of smallest order, while the determination for the Ree groups of characteristic three has been accomplished by J. H. Walter with the use of some deep theorems of modular character theory. However, our main tool is an elementary lemma of "Lie type" involving a very crude numerical estimate. In addition, we calculate the multiplicator for the smallest Suzuki group. Furthermore, preliminary investigations indicate that our methods might show that the multiplicators, or at least their 2-primary components, are trivial for the remaining family of Ree groups of characteristic two defined over $G F\left(2^{2 n+1}\right)$ for all $n \geqq N$, where $N$ is fairly small. Our lemma deals with a single automorphism; a suitable generalization of this result to a pair of commuting automorphisms would suffice to prove the preceding statement. Our main results are:

THEOREM 1. The Suzuki groups, with the exception of the one of smallest order, and the Ree groups of characteristic three have trivial multiplicators.

Theorem 2. The multiplicator of the smallest Suzuki group is elementary of order four. Furthermore, the multiplicator of its automorphism group is trivial.

With regard to notation, the commutator $x^{-1} y^{-1} x y$ of two elements of a group is denoted by $[x, y]$. The use of the Lie ring associated with a nilpotent group is standard [2]. Finally all groups mentioned are assumed to be finite.

The proof of Theorem 1 depends upon the following key lemma.

Received by the editors August 16, 1965.

1 This research was partially supported by National Science Foundation grants NSF GP 208 and GP-3701, respectively. 
Lemma. Let $G$ be a p-group of class at most $p+1$ whose commutator quotient group $G / G^{\prime}$ is elementary abelian of order $q=p^{n}$. Let $\alpha$ be an automorphism of $G$ of order $q-1$ which acts irreducibly on $G / G^{\prime}$. If $\alpha$ has a nonidentity fixed point in $G$, then $q=4,8$ or 9 .

Proof. Let $G=G_{1}, G_{2}, \cdots$ be the lower central series of $G$, so that by our hypothesis $G_{p+2}=1$. Since $G / G^{\prime}$ is elementary, it also follows that $G_{i} / G_{i+1}$ is elementary for all $i$. Let $L=\sum_{i=1}^{p+1} L_{i}$, where $L_{i}$ $=G_{i} / G_{i+1}$, be the Lie ring associated with the lower central series of $G$. In this case, $L$ is a Lie algebra over the field $F$ with $p$ elements and as is generally true of the associated Lie ring, $L$ is generated by $L_{1}$. Moreover, $\alpha$ induces an automorphism of this algebra, which we shall denote by the same letter, and $\alpha$ leaves each $L_{i}$ invariant. Furthermore, $\alpha$ is faithfully represented on $L_{1}=G / G^{\prime}$ by P. Hall's theorem [2, Theorem 12.2.2] as $p$ and $q-1$ are relatively prime. By assumption $\alpha$ acts irreducibly on $L_{1}$ and consequently the eigenvalues of $\alpha$ on $L_{1}$ in an algebraic closure of $F$ are $\lambda, \lambda^{p}, \cdots, \lambda^{p^{n-1}}$, where $\lambda$ is a primitive $(q-1)$ st root of unity. Since $L$ is a Lie algebra over $F$ and is generated by $L_{1}$, the eigenvalues of $\alpha$ on $L_{m}$ are of the form $\prod_{i=1}^{m} \lambda p^{a_{i}}$, where $0 \leqq a_{i} \leqq n-1$. Note that we may assume $n>1$, for otherwise $G / G^{\prime}$ would be cyclic and hence $G$ itself would be cyclic, in which case the lemma would hold trivially.

If $\lambda$ has a nontrivial fixed point on $G$, then it has a nonzero fixed point on some $L_{m}$, which implies that $\lambda$ has 1 as an eigenvalue on $L_{m}$. In this case, it follows that

$$
\sum_{i=1}^{m} p^{a_{i}} \equiv 0\left(p^{n}-1\right)
$$

for suitable integers $a_{i}, 0 \leqq a_{i} \leqq n-1$, and $1 \leqq m \leqq p+1$. Thus to prove the lemma, it will suffice to show that the above congruence implies that $q=p^{n}=4,8$ or 9 .

First of all, observe that

$$
\sum_{i=1}^{m} p^{a_{i}} \leqq(p+1) p^{n-1}<2\left(p^{n}-1\right)
$$

unless $q=4$. Thus either the lemma holds or our congruence implies the equality

$$
\sum_{i=1}^{m} p^{a_{i}}=p^{n}-1
$$

However, the right hand side of this equation is congruent to $p-1$ modulo $p$, thus forcing at least $p-1$ of the exponents $a_{i}$ to be 0 . In 
particular, $m \geqq p-1$ and since $n>1$, we have, in fact, $m>p-1$. Thus our equality reduces to either $(p-1)+p^{a}=p^{n}-1$ or $p-1+p^{a}+p^{b}$ $=p^{n}-1$, according as $m=p$ or $m=p+1$, where $0 \leqq a, b \leqq n-1$. The only solutions of these relations are $q=4$ or $q=4,8,9$ respectively, and the lemma is proved.

We now apply the lemma to prove Theorem 1. Let $S$ be one of the simple groups specified in Theorem 1. Properties of these groups are discussed in [4], [8] and [9]. In order to prove the theorem it will suffice to show that the only central extension of a cyclic group $Z_{p}$ of order $p$ by $S$ is a direct product. By a theorem of Gaschütz [2, Theorem 15.8.6], it is enough to prove that any central extension of $Z_{p}$ by $N(P)$, the normalizer in $S$ of a Sylow $p$-subgroup $P$ of $S$, is a direct product.

It is known that if $p$ is not the characteristic of $S$, then either $P$ is cyclic or $S$ is a Ree group, $p=2$, and $P$ is elementary abelian of order 8 . In the first case, $N(P)$ is a Frobenius group with kernel $C(P)$ and $|N(P): C(P)|=2,4$ or 6 , while in the latter case, $N(P)$ is of order 21.8 and $P$ is self-centralizing. In any of these cases it is trivial to verify the desired splitting. Thus the only case of any interest is that in which $p$ is the characteristic of $S$.

In this case, $P$ is of class $p$ and $P / P^{\prime}$ is elementary of order $q=p^{n}$, where $n \geqq 3$ and $p=3$ if $S$ is a Ree group and $n \geqq 5$ and $p=2$ if $S$ is a Suzuki group (since by assumption we are excluding the smallest Suzuki group). Furthermore, $N(P)=P A$, where $A$ is cyclic of order $q-1$ and acts faithfully and irreducibly on $P / P^{\prime}$. Let $E$ be a central extension of $Z_{p}$ by $P A$ and let $G$ be the unique Sylow $p$-subgroup of $E$, so that $G / Z_{p}$ is isomorphic to $P$. Then either $G / G^{\prime}$ is isomorphic to $P / P^{\prime}$ as an $A$-module or else $G / G^{\prime}$ has order $p^{n+1}$ and $Z_{p} \Phi G^{\prime}$.

Suppose first that $Z_{p} \nsubseteq G^{\prime}$. If $G / G^{\prime}$ is elementary, then $G$, and hence $E$, splits. If $G / G^{\prime}$ is not elementary, then the elements of order dividing $p$ in $G / G^{\prime}$ form an elementary $A$-invariant subgroup of order $p^{n}$ containing $Z_{p} G^{\prime} / G^{\prime}$. But $G / Z_{p} G^{\prime}$ is isomorphic to $P / P^{\prime}$ as an $A$ module. Since $A$ acts irreducibly on $P / P^{\prime}$, this is a contradiction. Thus $Z_{p} \subseteq G^{\prime}$. We conclude that a generator $\alpha$ of $A$ induces an automorphism of $G$ of order $q-1$ which acts irreducibly on $G / G^{\prime}$ and that $G / G^{\prime}$ is elementary of order $q$. Since $P$ has class at most $p, G$ has class at most $p+1$. Since $q>9$, the lemma applies to yield that $\alpha$ has no nontrivial fixed points on $G$, contrary to the fact that $\alpha$ fixes $Z_{p}$. This proves Theorem 1.

We turn now to the smallest Suzuki group and preserve the above notation. In this case, $p=2, n=3$ and $N(P)$ has the same structure as above. Furthermore, the multiplicator of $S$ is again a 2-group. 
Since $P$ is disjoint from its conjugates in $S$, it follows from [ 1 , Theorem XII, 10.1] $]^{2}$ that the multiplicator $H^{2}\left(S, C^{*}\right)$ of $S$ is isomorphic to the 2-primary component of the multiplicator of $N(P)$.

We shall first argue that this latter component has order at most four. To do this, it is sufficient to show that if $E$ is a central extension of a 2-group $B$ by $N(P)$ such that $B$ has no proper supplement in $E$, then $B$ has order at most four. It is clear that any such subgroup $B$ is contained in the Frattini subgroup of $G$, the unique Sylow $p$-subgroup of $E$. We first argue that $B \subseteq G^{\prime}$. If not, let $B_{0}$ be a maximal subgroup of $B$ containing $B \cap G^{\prime}$. Then the argument in the proof of Theorem 1 which shows that $Z_{p} \subseteq G^{\prime}$ can be repeated for $E / B_{0}$ to prove that $B / B_{0} \subseteq\left(G / B_{0}\right)^{\prime}$. Thus $B \subseteq G^{\prime} B_{0}$, so $B \subseteq\left(B \cap G^{\prime}\right) B_{0} \subseteq B_{0}$, a contradiction. Hence $G / G^{\prime}$ is isomorphic to $P / P^{\prime}$ and so is elementary of order 8 . Furthermore, since $P$ is of class $2, G$ is of class at most 3, and again the associated Lie ring $L=L_{1} \oplus L_{2} \oplus L_{3}$ is a Lie algebra over $F$. Moreover, a generator $\alpha$ of $A$ induces an automorphism of $L$ of order 7 , leaving each $L_{i}$ invariant and having eigenvalues $\lambda, \lambda^{2}, \lambda^{4}$ on $L_{1}$, where $\lambda$ is a primitive seventh root of unity. Thus the eigenvalues of $\alpha$ on $L_{2}$ are among $\lambda^{3}, \lambda^{5}, \lambda^{6}$, so that, in particular, $\alpha$ has no nontrivial fixed point on $L_{2}$. This implies that $B=L_{3}$.

We extend $L$ to a Lie algebra over $F(\lambda)$, which we still denote by $L$, and choose a basis $x, y, z$ of $L_{1}$ consisting of eigenvectors for the eigenvalues $\lambda, \lambda^{2}, \lambda^{4}$ of $\alpha$ respectively. Using the defining relations for a Lie algebra, it follows that $L_{3}$ (which is $L^{3}$ ) is spanned by the eight vectors $[x y z],[y z x]$, and all $[u v u]$, where $u$ and $v$ run over all distinct pairs among $x, y$ and $z$. Since $\alpha$ fixes $L_{3}$ elementwise, these last six vectors must be 0 , for otherwise $\alpha$ would have an eigenvalue distinct from 1 on $L_{3}$. We conclude that $L_{3}$ is at most of dimension 2 and hence that $B$ has order at most 4 .

In view of the result of Cartan-Eilenberg stated above, it is therefore enough to prove Theorem 2 to exhibit a central extension $E$ of $B$ by $N(P)$ with the required properties in which $B$ is elementary of order 4 . A group $E$ satisfying these conditions is defined by generators $y, x_{i}, 1 \leqq i \leqq 3$ and relations: ${ }^{3}$

$$
\begin{gathered}
x_{1}^{2}=x_{23} x_{122}, \quad x_{2}^{2}=x_{13} x_{23} x_{121} x_{122}, \quad x_{3}^{2}=x_{12} x_{13} x_{23}, \\
x_{131}=x_{122}, x_{133}=x_{121} x_{122}, \quad x_{232}=x_{121}, x_{233}=x_{122}, x_{123}=x_{121}, x_{231}=1, \\
y^{7}=1, \quad x_{1}^{y}=x_{2}, \quad x_{2}^{y}=x_{3}, \quad x_{3}^{y}=x_{1} x_{2},
\end{gathered}
$$

2 The applicability of this result was pointed out to us by R. Swan.

3 For simplicity we set $x_{i j}=\left[x_{i}, x_{j}\right], x_{i j k}=\left[x_{i}, x_{j}, x_{k}\right]$. 
the derived group of the group of $\left\langle x_{1}, x_{2}, x_{3}\right\rangle$ is of exponent two and $\left\langle x_{1}, x_{2}, x_{3}\right\rangle$ is of class at most three.

In this group $E$ we choose $B=\left\langle x_{121}, x_{122}\right\rangle, G=\left\langle x_{1}, x_{2}, x_{3}\right\rangle$ and $A=\langle y\rangle$.

Finally let $A$ be the automorphism group of $S$, the smallest Suzuki group. By Theorem 11 of [8], $|A: S|=3$, so that a Sylow 3subgroup of $A$ is cyclic of order 3. Thus the Sylow subgroups of $A$ are cyclic for all odd $p$ and so the multiplicator of $A$ is a 2-group by a well-known result of Schur. It follows therefore as with $S$ that the multiplicator of $A$ is elementary of order at most 4 .

We refer once again to the Lie algebra $L$ over $F(\lambda)$ and the automorphism $\alpha$ of $L$ of order 7 and preserve the above notation. In $A$ there exists an element $\beta$ of order 3 such that $\alpha^{\beta}=\alpha^{4}$. We consider the action of the group $\langle\alpha, \beta\rangle$ on $L$. To show that $A$ has a trivial multiplicator, it will suffice to prove that $\langle\alpha, \beta\rangle$ has no fixed points on $L_{3}$. By the preceding analysis, it will be enough to show that $\beta$ has no fixed points on the space spanned by $[x y z]$ and $[y z x]$. Since the eigenspaces of $\alpha$ on $L_{1}$ are one-dimensional, $\beta$ must permute them, and so we can choose the basis $x, y, z$ of $L_{1}$ with $x^{\beta}=y, y^{\beta}=z$, and $z^{\beta}=x$. But then $\beta$ transforms $[x y z]$ and $[y z x]$ into $[y z x]$ and $[z x y]=[x y z]$ $+[y z x]$ respectively, from which the desired conclusion follows at once. This completes the proof of Theorem 2.

\section{BIBLIOGRAPHY}

1. H. Cartan and S. Eilenberg, Homological algebra, Princeton Univ. Press, Princeton, N. J., 1956.

2. M. Hall, The theory of groups, Macmillan, New York, 1959.

3. Z. Janko, $A$ new finite simple group with abelian 2-Sylow subgroups and its characterization (to appear).

4. R. Ree, A family of simple groups associated with the simple Lie algebra of type $\left(G_{2}\right)$, Amer. J. Math. 83 (1961), 432-462.

5. - A family of simple groups associated with the simple Lie algebra of type $\left(F_{4}\right)$, Amer. J. Math. 83 (1961), 401-420.

6. I. Schur, Über die Darstellung der symmetrischen und der alternienden Gruppe durche gebrochene lineare Substitutionen, J. Reine Angew. Math. 139 (1911), 155-250.

7. R. Steinberg, (to appear).

8. M. Suzuki, On a class of doubly transitive groups, Ann. of Math. 75 (1962), 105145 .

9. H. N. Ward, On Ree's series of simple groups, Trans. Amer. Math. Soc. (to appear).

University of Chicago and NORTHEASTERN UNIVERSITY 